\title{
DIRECTIONAL COARSENING OF NICKEL BASED SUPERALLOYS : DRIVING FORCE AND KINETICS
}

\author{
Muriel Véron, Yves Bréchet and François Louchet \\ Groupe Physique du Métal, LTPCM-CNRS (URA 29) \\ BP 74, 38402 St Martin d'llères
}

\begin{abstract}
:
The $\gamma^{\prime}$ morphology of a single crystal superalloy, that had first undergone a plastic prestrain, has been studied after having been annealed at high temperature. Observation of a directionaly coarsened microstructure has revealed the dominant role of plasticity, more precisely of dislocations at matrix/precipitates interfaces, in the driving force of directional coarsening. A model and a 3D computeur simulation for the phenomenon has been developed, based on a model that take into account plasticity induced during creep and misfit stresses. Results of the model are presented and compared to experimental observations on AM1 superalloy. Morphology maps describing the expected rafting geometry for other superalloys (other misfits) under a given applied stress are produced. Finally, considerations on the mechanical properties of the coarsened microstructure, in particular the ability of a dislocation to glide in the new morphology, are discussed.
\end{abstract}

\section{Introduction :}

Directional coarsening or "rafting" of $\gamma^{\prime}$ precipitates in single crystal Nickel based superalloys is usually observed to take place under stress at high temperatures (typically $1050^{\circ} \mathrm{C}$ ). Though it has becn extensively studied experimentally $[1,2,3$ for example $]$ and theoretically $[4,5,6]$, the exact nature of the driving force and the associated kinetics are still a matter of controversy. It is generally agreed that the rafting morphology and the kinetics are ruled by two key parameters, namely the misfit between the two phases, and the amplitude and sign of the uniaxial applied stress. Though most of the previous investigations were based on the elastic incompatibilities between the two phases, plasticity occurs in the stress-temperature domain where directional coarsening is observed $[7,8]$. Since plastic deformation is driven by the elastic energy stored in the material, it can participate significantly to its relaxation, and has to be taken into account in the modelling of directional coarsening.
The first part of the present contribution reports an experimental investigation of the possible role of plasticity in rafting mechanisms: the influence of a prestrain on morphological evolution during annealing of a superalloy has been put forward. Then the second part is devoted to the physical basis and the results of a computer simulation, taking the local plastic strain as the origin of the main driving force for directional coarsening.

Finally, some properties of the coarsened structures after different creep-simulated times are discussed.

\section{Driving force}

Since dislocations have been observed at the matrix/precipitate interfaces in specimens tested under service conditions, we have investigated the influence of a pre-strain on the phenomenon. We have studied the morphological evolution during annealing of a prestrained sample (in compression) of AM1 superalloys [9], provided by SNECMA.

The initial microstructure of this alloy is two-phased : $\gamma^{\prime}$ precipitates, of an average size of $0.45 \mu \mathrm{m}$ and with a volume fraction about $70 \%$ at room temperature, are well aligned in a $\gamma$ matrix. The average distance between two precipitates -i.e. the size of matrix channel- was determined using image analysis, and is about $0.09 \mu \mathrm{m}$. The misfit is defined as $\delta=2\left(a \gamma^{\prime}-a \gamma\right) /\left(a \gamma^{\prime}+a \gamma\right)$, where ay and a $\gamma^{\prime}$ are respectively the lattice parameters of the $\gamma$ and $\gamma^{\prime}$ phases. Its value was determined to be $-3 \times 10^{-3}$ at $1050^{\circ}$ $\mathrm{C}[10]$.

\section{Experimental procedure}

Two compression samples were selected : both were introduced in a furnace and left 1 hour at $850^{\circ} \mathrm{C}$ $(1123 \mathrm{~K})$. At this temperature the kinetics of coarsening are very low. After stabilisation, sample (b) was stressed in compression at $450 \mathrm{MPa}$ up to a strain of about $1 \%$ (then the stress was removed), whereas sample (a) was left without stress. Then the temperature was increased, and both samples were left at $1323 \mathrm{~K}$ during $20 \mathrm{~h}$.

The only difference between the two specimens is that sample (b) has undergone a strain of about $1 \%$ whereas 
sample (a) was not strained. Then both samples were further heat treated, without any load, at $1323 \mathrm{~K}$ during 20 hours and then air cooled down to room temperature.

Faces parallel to the various (100) directions (parallel or perpendicular to the stress axis ) were mechanically polished, then etched to reveal the two phases. The microstructure was then observed in a scanning electronic microscope (SEM) (Jeol 6400). Thin foils have been also prepared for TEM observations of the interfaces.

\section{Results and discussion.}

TEM observations on sample (a) evidence a high density of dislocations, in the matrix corridors parallel to the stress axis. The corridors perpendicular to the stress axis are nearly dislocation free. Sample (b) shows no evidence of plastic deformation.

SEM observations have revealed differences in microstructures between the two samples. Though both of them were aged without applied stress at 1323K, sample (a) (which was not prestrained) has no sign of directional coarsening, the precipitates are still cuboidal with a size of about $0.45 \mu \mathrm{m}$; whereas sample (b) which was prestrained shows pronounced directional coarsening : the precipitates are elongated into rafts of $3 \mu \mathrm{m}$ length in the (001) directions containing the compression axis (see fig. 1).

In absence of an externally applied stress, simply by prestraining the sample, directional coarsening was observed.
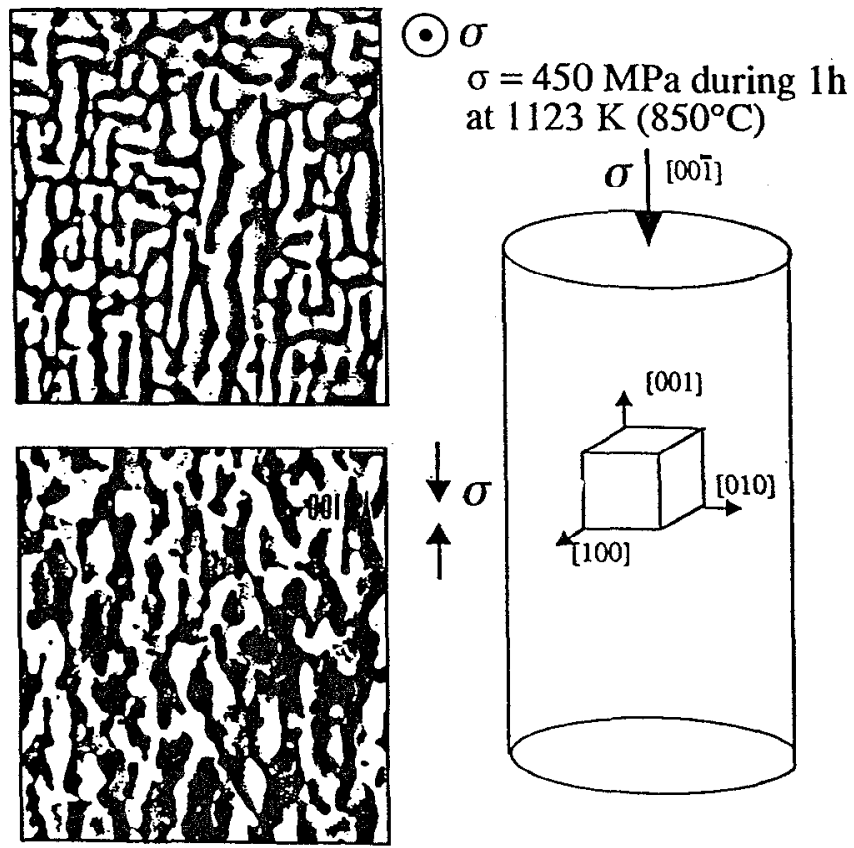

(b)

Figure 1:a) Microstructure of the prestrained sample after annealing. The observations are made in two directions : perpendicular (first image) and parallel to the stress (at the bottom). b) Microstructure of the "non strain" sample, observed in the similar directions.

This results suggest that the dislocations, introduced during the prestrain and forming networks on specific interfaces as observed in TEM, have an effect on the matrix/precipitates interfaces, that is therefore probably to relax selectively the misfit stresses in the matrix corridors (the ones parallel to the applied stress in the example considered). This effect was already suggested for the dislocations networks observed during creep $[7,11,12]$, but it had yet not been clearly separated from the effect of the applied stress.
As the net effect of prestrain is to relax the internal stresses only in some channels of the $\gamma$ phase, coarsening is then more likely to be associated with inhomogeneous relaxation of internal stresses rather than to the coupling between the applied stress and the misfit stress. This experiment means clearly that the driving force due to plastic deformation is dominant as compared to other effects. A model for directional coarsening can be proposed : this anisotropic relaxation of coherency stresses induces a gradient in chemical potential (due to a gradient in elastic energy) which drives the diffusive flow 
through the channels, responsible for directional coarsening (fig. 2).

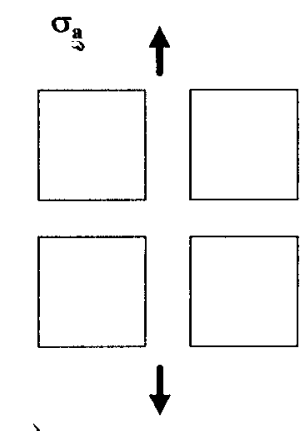

a)

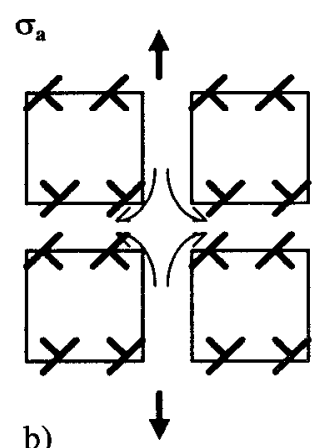

b)

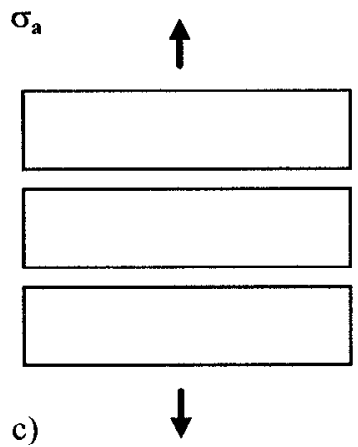

Figure $2:$ a) In the initial condition, the misfit is isotropic on the $\gamma / \gamma^{\prime}$ interfaces. b) Under stress at high temperature, dislocations have developed and they relax selectively some $\gamma / \gamma^{\prime}$ interfaces. A diffusional flow occurs in the matrix (probably also in the precipitates), and c) the microstructure evolves.

A similar experiment has been conducted for a pre-strain in tension along a $<001>$ direction, and the same observations were made : directional coarsening, with rafts perpendicular to the 'pre-strain axis', was observed [13].

\section{Directional coarsening modelling}

\section{Physical basis}

Due to the combination of misfit and applied stresses, the different types of $\gamma$ phase channels are differently stressed. Under tension for instance, and in the case of a negative mistit, the "horizontal" channels (i.e. perpendicular to the stress axis) undergo a larger shear stress than the "vertical ones" (which contain the stress axis). As a consequence, both plastic flow and relaxation of misfit stresscs are expceted to take place first in horizontal channels, which is confirmed by transmission electron microscopy of dislocations at $\gamma / \gamma$ interfaces in these channels. A driving force therefore arises for diffusion between horizontal (relaxed) and vertical (non relaxed) channels. This driving force is obviously still present if the sample has been prestrained, and subsequently aged at high temperature without any applied stress, as reported in the first part.

The previous results allowed us to put forward a driving force for directional coarsening in the plastic regime. We have then modelled the phenomenon and developed a computer simulation taking into account the local plastic strain as the main driving force for coarsening. The simulation is at a mesoscopic scale, thus it provides the evolution of the entire precipitate population in the matrix, and the results can be directly compared to SEM images. Details of the simulation have been published elsewhere [14], in this contribution, we will present now only the main steps of the program, but some equations will be developed the Appendix.

First, the microstructure we have chosen to simulate consists of a network of matrix channels (not of precipitates), because the matrix undergoes much of the microstructure evolution and parameters such as the width of the channels is pertinent for directional coarsening. This network is a simple cubic lattice with a lattice constant of the average ( $\gamma$ channel plus $\gamma^{\prime}$ precipitate) size. The width of these channels is distributed according to an experimental distribution measured on SEM pictures of the AM1 alloy.

The principle of the simulation consists in calculating the flux of matter between the different matrix channels, due either to plastic flow, or to diffusional flow resulting from a chemical potential gradient. The different fluxes added together will lead too a redistribution of matter between the different channels (with a constant total volume fraction of precipitates) and to directional coarsening. In order to do so, we need to evaluate the local stresses inside each channel. As the value of the local stress in a channel depends on the width of this channel, the flux of matter is calculated for small time steps, then the width distribution is reset, and the new local stresses are reestimated in all the corridors. Time steps were chosen to be small enough compare to the typical coarsening time.

Two stresses contribute to the local stress : on one hand the coherency stresses (misfit stresses) which may be relaxed on some interfaces due to the presence of dislocations; and on the other hand, the macroscopic applied stress, which is distributed between the channels and the precipitates according to a composite effect. The stresses in a given channel also change the local chemical potential of the elements in a channel and therefore a difference in local stresses in neighboring channels will result in a cross-diffusion flux contributing to the channel width evolution (this was observed in the prestrain sample experiment). This local stress in each channel also leads to plastic flow (and thus to mass transport from one channel to its neighbors). 
In order to compute the local stresses induced by the applied stress in the channels, we have represented the microstructure as a complex composite structure. The equilibrium laws that govern this system are the partitioning of stresses between $\gamma$ and $\gamma^{\prime}$ phases and the compatibility of strain rates of the matrix in the vertical and horizontal channels, assuming the $\gamma^{\prime}$ phase does not deform plastically.

From this two equations and from the relation between the strain rate and the stress in the matrix, the local stresses $\sigma * \mathrm{v}$ and $\sigma * \mathrm{~h}$ in the vertical and horizontal channels respectively can be computed as functions of the applied stress and the coherency stresses, in each corridors, at each time step. The values of the coherency stresses can be tuned to account for the relaxation process. Thus in the "relaxed" channels (horizontal in our example), the value of the stresses is zero, and in the "non relaxed" ones (vertical), they remain at their initial value.

As a result of this partial relaxation, effective stresses in tensile creep are :

$$
\begin{gathered}
\sigma_{v}^{*}=\left(\frac{w_{h}}{1}\right)^{\frac{1}{n}} \frac{\sigma_{a}\left(1+w_{v}\right)^{2}-E_{v} \delta_{v}\left(21+w_{v}\right)}{\left(\frac{w_{h}}{1}\right)^{\frac{1}{n}}\left(21+w_{v}\right) v_{v}+1^{2}} E_{v} \delta_{v} \\
\sigma_{h}^{*}=\frac{\sigma_{a}\left(1+w_{h}\right)^{2}-E_{v} \delta_{v}\left(21+w_{h}\right.}{\left(\frac{w_{h}}{l}\right)^{\frac{1}{n}}\left(21+w_{h}\right) w h+l^{2}}
\end{gathered}
$$

where 1 is the mean size of a precipitate, $w_{h}$ and $w_{v}$ are respectively the widths for the horizontal and vertical channels, $\sigma_{a}$ is the applied stress intensity, $E_{v}$ is the Young modulus of the composite structure and $\delta_{\mathrm{y}}$ is the misfit at the vertical interfaces.

Once the stresses are determined in each channel, the next step of the program is to calculate the corresponding elastic energy densities.

In addition to this bulk term, another driving force stems from the capillary effect : the thinner channels tend to disappear and the thicker tend to grow, irrespectively of their orientation, simply to reduce the total surface in a process similar to Oswald's ripening.

Finally, in a given channel (i), the total energy density $E_{\text {tot }}(i)$ is :

$$
E_{\text {tot }}(i)=E_{\text {el }}+E_{i} \text {. }
$$

where $E_{e l}$ is the elastic energy density stored in the channel due to the local stress, and $E_{i}$ is the interface energy density.

The inhomogeneities in these energy densities will drive a diffusive flux between the channels, particularly between the vertical and horizontal channels for whom the elastic energy densities are different. The driving force carrying atoms from a given channel (i) to an other channel (j) is the chemical potential gradient which can be approximated by :

$$
\nabla \mu(\mathrm{ij})=\left(\mathrm{E}_{\text {tot }}(\mathrm{j})-\mathrm{E}_{\text {tot }}(\mathrm{i})\right) / 1
$$

The characteristic distance 1 is of the order of precipitate size.

For a given element, the mobility $M$ is related to the diffusion coefficient by the Einstein relation : $M=D / k T$, where $\mathrm{D}$ is the diffusion coefficient of a given element. As diffusion coefficients values are not accurate enough to make differences between diffusion coefficients of nickel, aluminium or chromium in $\gamma$ phase which are the major elements of the alloy, D can be taken as an average diffision coefficient. The response to the driving force gives the diffusional flux $\mathbf{J}_{\mathbf{i j}}$ :

$J_{\mathrm{ij}}=-\mathrm{D} / \mathrm{kT} \nabla \mu(\mathrm{ij})=-\mathrm{D} / \mathrm{kT}\left\{\left[\mathrm{E}_{\text {tot }}(\mathrm{j})-\mathrm{E}_{\text {tot }}(\mathrm{i})\right] / \mathrm{I}\right\} \mathrm{xc}$

where $c$ is the concentration of a given element [15].

After this step, we can calculate what is the algebraic change in channel width, per time step. For a given diffusional flux $\mathrm{J}_{\mathrm{ij}}$, the change in channel width depends on the surface $S$ of the channel cross-section. Since a channel has 12 neighbors, the contributions of each flux have to be added and the width variation of channel (i) due to diffusion is :

$$
W_{\mathrm{d}}=\Omega / \mathrm{I}^{2} \sum_{12 \text { neighbors }} \mathrm{J}_{\mathrm{ij}} \mathrm{S}_{\mathrm{ij}}
$$

where $\Omega$ is the atomic volume, and $\mathrm{Sij}$ is the surface through which the diffusional flux goes. For Sij we choose the cross section of the narrowest channel (i) or (j).

Direct plastic flow (transport of matter by dislocations) also contribute to the evolution of channel width. The plastic rate in both types of corridors is : $\varepsilon=\mathrm{K}\left(\sigma^{*}\right)^{\mathrm{n}}$, where $\sigma^{*}$ is the local stress. 
Then the channels width variation rate due to plastic flow is :

$$
\begin{aligned}
& w p, v=K\left(\sigma_{v}^{*}\right) \frac{w_{v}}{l} \text { for vertical channels } \\
& w h, v=K\left(\sigma_{v}^{*}\right)^{n} w_{h} \text { for horizontal channels }
\end{aligned}
$$

The superposition of the contribution of plastic flow of this channel with the mass transfer with neighboring channels by diffusion.:

$$
\dot{w}=\dot{w} p+\dot{w d}
$$

The rate of evolution of a channel width is calculated for the microstructure at this level of evolution. After the microstructure has changed, local stresses have changed and new rates are calculated.

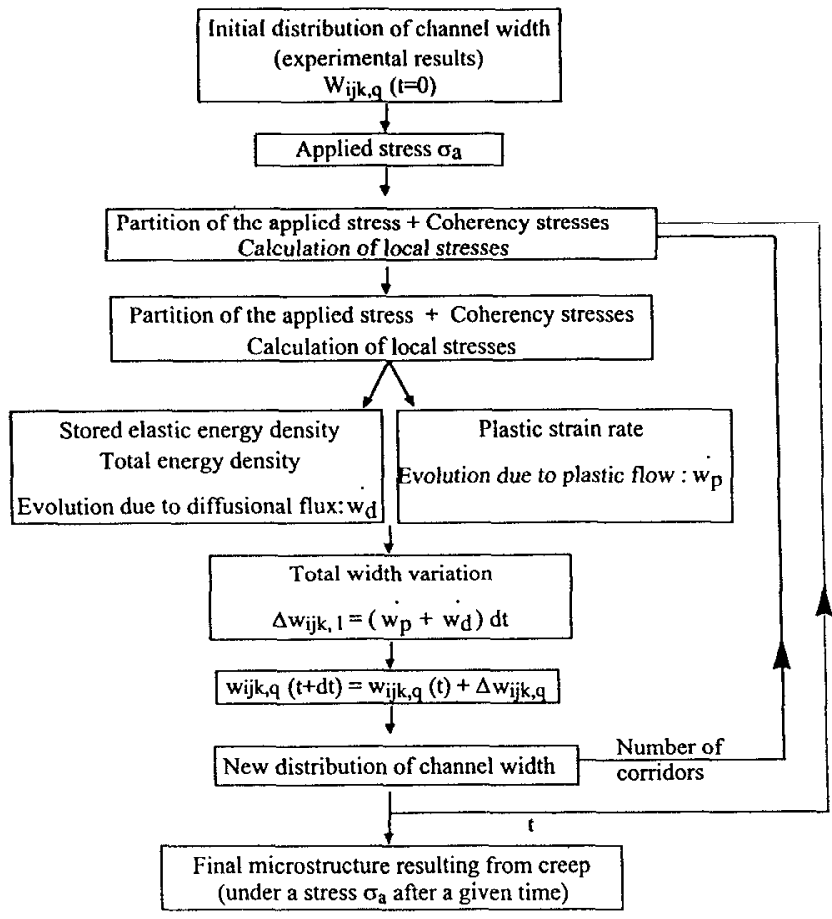

Figure 3 : Flow Chart of the simulation. The system size is typically $15 \times 15 \times 15$ precipitates (10125 channels). The boundary conditions are periodic. Each channel is characterised by three indices (ijk) for its position, one index (q) for its type (vertical (v) or horizontal (h)) and its width $w_{i j k}, q$
These local rules of evolution have been implemented in the computer simulation in order to describe the coupled evolution of a population of channels.

The flow chart of the program is presented in figure 3. At each time step, we calculate the variation in width of each channel according to equation (9). We then compute the new width distribution and the associated stress states which will allow to compute at the next step the new rate of evolution of channels. The iteration of this procedure allows us to compute the morphological evolution of the system with a computer time which is unambiguously related to the physical time since our evolution equations are fully deterministic.

The value of the constants (see Table 1) used were found in literature $[10,11,16]$, provided by SNECMA (Norton's coefficient for AM1 in tensile creep) or are own results (Norton's coefficient for AM1 in compressive creep, width distribution of channels).

\begin{tabular}{|l|l|}
\hline Volume fraction [10]: & $\mathrm{f}_{\mathrm{v}}=50 \%$ at $1323 \mathrm{~K}$ \\
Elastic constants: & $\mathrm{E} \gamma=70 \mathrm{Gpa}$ \\
& $\mathrm{E} \gamma=90 \mathrm{Gpa}$ \\
Creep parameters : & $\mathrm{n}_{\mathrm{t}}=5$ (tension), \\
(Norton's law coefficients) & $\mathrm{n}_{\mathrm{c}}=2.8$ (comp.) \\
Interface energy [16]: & $\Gamma=20 \mathrm{~mJ} \cdot \mathrm{m}^{-2}$ \\
Diffusion coefficient in $\gamma[11]:$ & $\mathrm{D}=10^{-14} \mathrm{~m}^{2} . \mathrm{s}^{-1}$ \\
Mean channel width : & $0.095 \mu \mathrm{m}$ \\
Mean channel length & $0.45 \mu \mathrm{m}$ \\
\hline
\end{tabular}

Table 1: Data used in the simulation

$\underline{\text { Results }}$

\section{Morphology maps :}

Simulation has been run to study the behaviour of AM1 superalloy under different stress conditions. The results, both on the predicted morphologies and kinetics of the evolution, are in good agreement with experimental results (fig. 4). For tensile and compressive stresses, we predict coarsening in opposite directions as observed experimentally.

Moreover the first coarsening event is predicted to occur after about $5 \mathrm{~h}$ as observed experimentally in this range of stress. Thus it has been developed to predict the behaviour of other superalloys, i.e. superalloys with other misfit values (other parameters, such as elastic constants, or creep exponents, have been kept constant in order to simplify the calculation and also because they were not available for all cases). As a result, the predictions give a qualitative idea of the evolution of microstructures under stress at high temperature. These results are presented in a morphology map (fig. 5), that shows -as a function of 
the applied stress $\sigma$ and the misfit value $\delta$ - what will be the microstructure of a given alloy after $20 \mathrm{~h}$ of creep.
The map is devised in regions, in which the plastic and diffusive fluxes can either contribute to the same type of evolution or to opposite ones.

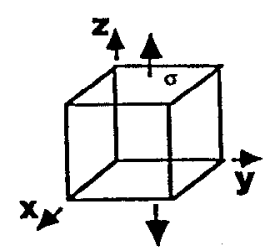

$-0.4 \mu \mathrm{m}$

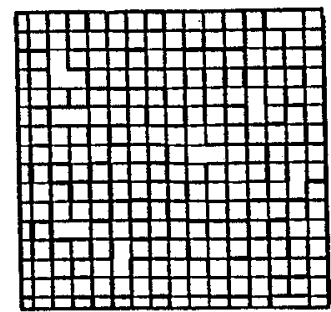

(a)

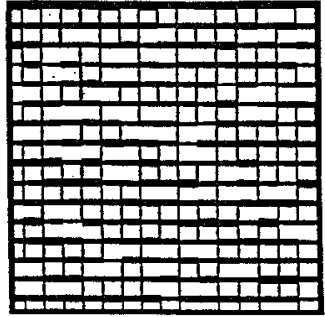

(b)

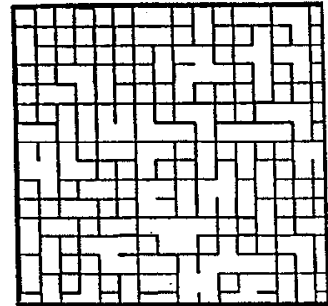

Figure $4:$ Morphologies o
20h of compressive creep
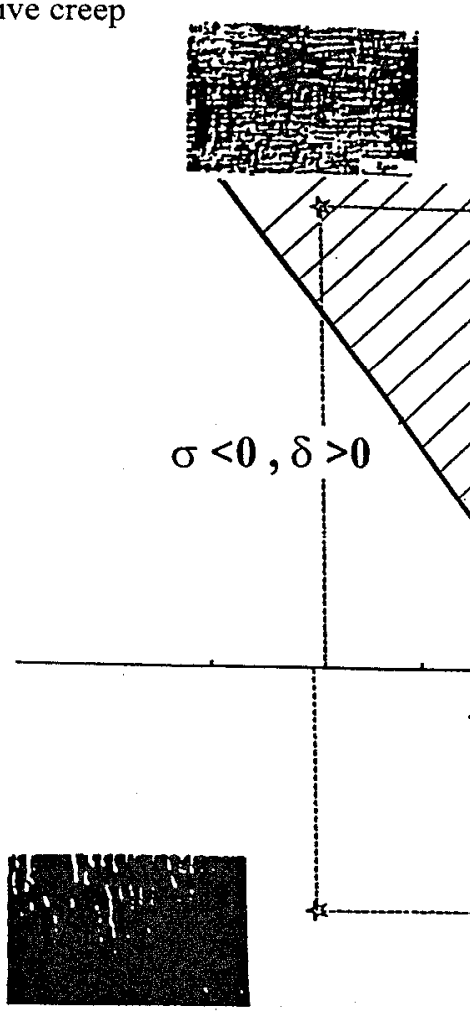

(e)

(d)
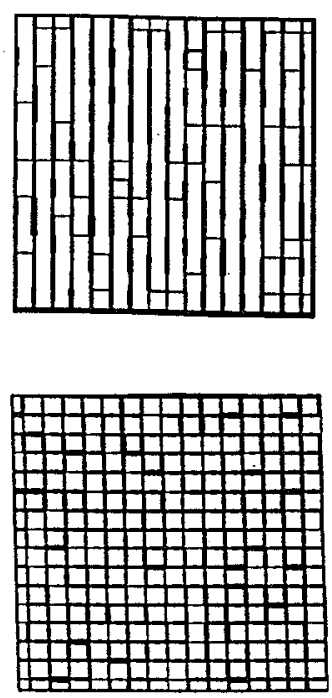

e)

\& (c) after $10 \mathrm{~h}$ of tensile creep, (d) \& (e) after 
When those two contributions where competing, we have determine when one of them became dominant, and we assume that the microstructure will evolve in the direction of this contribution. In particular, for alloys with positive misfit the fluxes were opposite : for example in tension, the plastic flow would have tend to lead to a coarsening perpendicular to the stress axis, but the diffusive one to rafts parallel to the axis. We have then determine for what value of stress the plastic contribution would overcome the diffusive one : this line has been drawn to be the limit between two different modes of coarsening. As a result, two behaviours are predicted for alloys with positive misfit, in relation with the intensity of the applied stress. Some regions of the map have been validated with experiments found in literature, but some other characterisations should be done, especially for alloys with positive misfit : when the competition between the driving forces exists, the results suggests the existence of a region of isotropic coarsening.

\section{Mechanical properties of the coarsened structures :}

In superalloys, the yield stress is due to three mechanism: hardening by solid solution (mainly in the $\gamma$ phase), precipitation hardening (high volume fraction of $\gamma^{\text {' }}$ precipitates), and internal stresses (namely coherency stresses). The contribution to the critical resolved shear stress of both solid solution hardening and $\gamma^{\prime}$ precipitation have been investigated in alloys with a low volume fraction of precipitates $\left(f_{v}<50 \%\right)$ and no strong anisotropic coherency effects [17]. In this study, we will only consider the contribution of the precipitation of a coherent, harder phase in high volume fraction $\left(f_{v}>\right.$ $50 \%$ ). Moreover, we will concentrate in the regime where precipitates are circumvented. This regime corresponds to the stationnary creep observed at high temperature in commercial superalloys [18], when the Orowan process operates. Since the simulation provides a $3 \mathrm{D}$ matrix channel structure, it is a good tool to estimate the evolution of the matrix channel width in the <111> planes, and therefore to make some prediction on the evolution of the yield stress when the microstructure becomes rafted.

When loaded in the [001] direction (the main direction of stress during service), the four $\langle 111\rangle$ slip planes are similarly activated in the single crystal. To take into account the microstructure effect on some mechanical properties of the alloy, we have investigated what is the stress for a dislocation to go through a matrix corridors, in one of this four planes. Therefore we have taken cuts of the structure corresponding to different coarsening time, in the $<111>$ directions (fig. 6).
In the cuts corresponding to the initial state, the geometry of precipitates depends strongly on the chosen level of the cut : if the level of cut corresponds to the middle of the precipitates, the precipitates are mostly hexagonal with acute angles (fig. 6.a); but if it corresponds to $3 / 4$ of the precipitates, then in the slip planes, they have triangular shapes, with obtuse angles (fig. 6.b). Thus the behaviour of dislocations can be expected to be different, especially concerning the shearing of precipitates [19], and this is to take into account for plastic localisation [20]. On the contrary, after directional coarsening is completed, no such differences are observed in cuts.
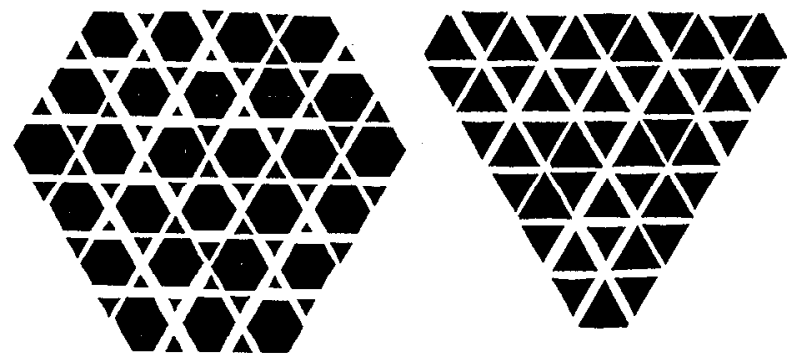

(a)

(b)
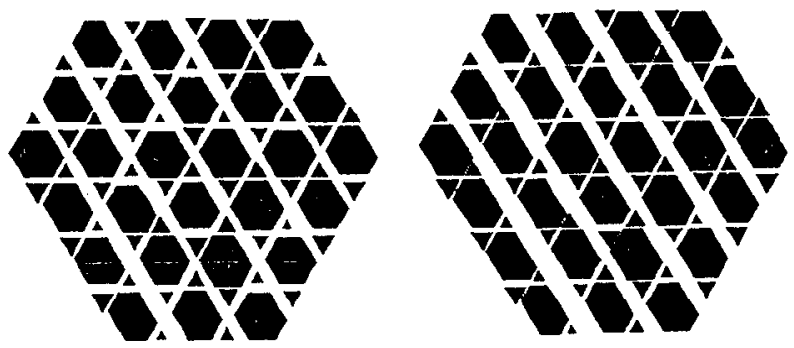

(c)

(d)
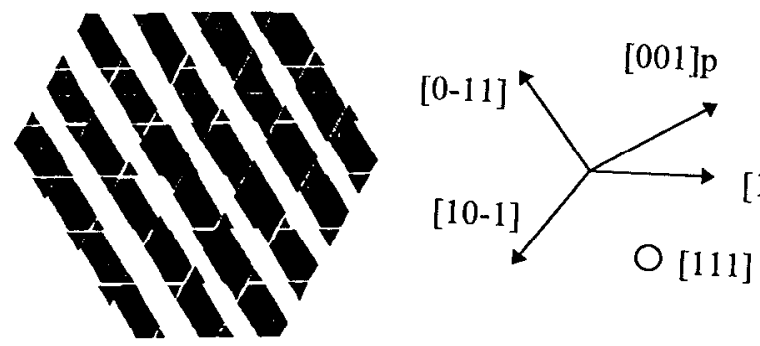

[1-10]

(e)

Figure 6 : Cuts of the simulated $\gamma / \gamma^{\prime}$ network in the $\{111\}$ plane : (a) initial state, cut realized in the middle of the precipitates, (b) initial state, cut realized near an edge of the precipitates, (c) after $5 \mathrm{~h}$ under stress at $1050^{\circ} \mathrm{C}$, (d) after $10 \mathrm{~h}$ and (e) $20 \mathrm{~h}$ in the same conditions.

As far as the Orowan process is concerned, the behaviour of dislocations is dependant on the obstacles volume fraction and on the distance between obstacles. The stress required for a dislocation to circumvent obstacles, is given by : 


$$
\tau_{\mathrm{or}}=\mu \mathrm{b} / \mathrm{L}
$$

where $\tau_{\text {or }}$ is the Orowan stress, $\mu$ is the shear modulus, $b$, the Burgers vector of the dislocation, and $\mathrm{L}$, the distance between the obstacles.

In superalloys, the distance between precipitates is then of crucial importance : for a given applied stress, dislocations will pass through matrix channels only if their width is large enough.

We have run the simulation to calculate the channel width evolution with time, in the gliding plane for a stress along [001]. The results are presented in figure 7.a, which shows the size of the matrix channels as a function of time, distinguishing the ones who are thinning (lype 1 and 3 , the vertical ones) and the ones who are thickening (type 2, the horizontal ones).
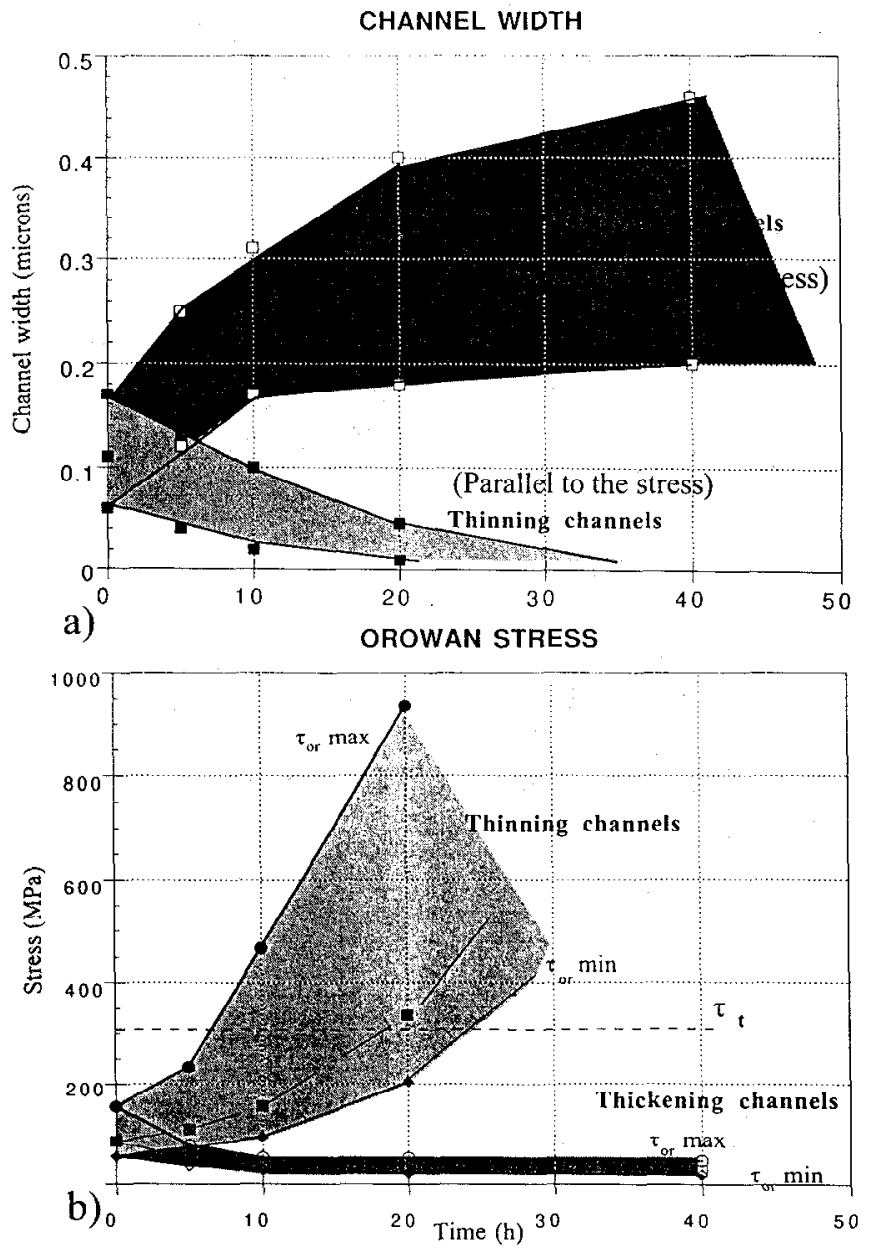

Figure 7 : (a) Channel width evolution versus time (b) Orowan stress evolution versus time. $\tau_{1}$ is an estimation (FEM calculation) of the effective stress in the channels, taking into account coherency stresses, for an applied stress as high as $300 \mathrm{MPa}$.
On this graph, the extrema and average of the distribution width of the channels are presented. First, the microstructure seems to evolve fast, and then, the kinetics become slower. We calculated that after $20 \mathrm{~h}$ under a tensile stress of $120 \mathrm{MPa}$, the structure is almost completely rafted - the "vertical" channels have disappeared, and no further strong evolution is observed.

We have then estimated at a temperature of $1050^{\circ} \mathrm{C}$ what was the evolution of the Orowan stress when the microstructure is evolving (fig. 7.b).

For the channels which are thickening, the Orowan stress is decreasing and it reaches a plateau when the width is stabilising. On the contrary and as expected, it increases for the thinning channels, and very fast, it reaches prohibitive values. For example, after 10 hours, some channels are smaller than $20 \mathrm{~nm}$, and the theoritical Orowan stress is then $470 \mathrm{MPa}$

For an applied stress of the range of $120 \mathrm{MPa}$, even with the contribution of coherence stresses, this level of stress will not been achieved in this channel, and no dislocation will be able to overpass it. In figure 7 , we have indicated the value of the effective stress in the vertical channels for an applied stress of $300 \mathrm{MPa}$, it is about $100 \mathrm{MPa}$. As a result, after a couple of hours, the fraction of penetrable channels is strongly reduced.

After typically 20 hours, dislocations are gliding mainly in the horizontal channels between two $\gamma^{\prime}$ rafts, each dislocation building and contributing to a back-stress for the new one.

The bypassing mechanism of the precipitates at $1050^{\circ} \mathrm{C}$ combines glide and climb process. Howcver, the strong aspect ratio of the rafted preeipitates will tend to prevent the climb of dislocations. Then when the rafts are well formed, glide is the main deformation mecanism. Therefore to propagate a dislocation into the rafted microstructure, dilsocations have now to shear the precipitates. As some shearing events have been reported in litterature in a similar alloy after $20 \mathrm{~h}$ of creep [18], the stress in the horizontal channels must increase and reach the shear stress of the precipitates.

Since the shearing process of the ordered intermetallic phase involves pairs of matrix $a / 2<110>$ dislocations coupled with an anti-phase boundary (APB), a better knowledge of the APB's energy in the system would be useful to study this phenomenon. It would then be possible to simulate the occurrence of the shearing process, and to study a coupling of the shearing of rafts with the change of connectivity of the phases. 


\section{Conclusion}

With a simple experiment, we were able to put forward the crucial role of dislocations in the directional coarsening process. Dislocations are at the origin of an anisotropy in elastic energy in different types of channels with respect to the applied stress. This anisotropy drives a diffusion flux, responsible for the microstructural evolution. The directional coarsening process has been simulated, and the results are in a good agreement with experimental observations. Moreover, it has been possible to predict the rafting behaviour of others superalloys under different conditions. particularly, a possible isotropic coarsening behaviour for alloys with a positive misfit has been predicted, due to the competition of the difference flows driving the morphological evolution. With simple microstructural considerations, based on the $\gamma / \gamma^{\prime}$ patterns provided by the simulation, some preliminary investigations have been conducted to study the deformation mechanism. With a better knowledge of the shear stress in the commercial superalloys, it will be possible to calculate as a function of time, temperature and applied stress, when the shearing of the rafts occurs. The 3-D simulation we have developed appears as a very promising tool to study high temperature mechanical properties of superalloys, with respect to the complex evolution of the microstructure.

\section{Acknowledgements :}

This work was supported by the French CPR "SSM" of CNRS. The author would like to thank SNECMA for providing the material, and for supporting this work through a research grant (Muriel Véron). We also would like to thank the french GDR "Simulation numérique" for support.

\section{References:}

[1] J.K. Tien and S. M. Copley, "The Effect of Uniaxial Stress on the Periodic Morphology of Coherent Gamma Prime Precipitates in Nickel-base Superalloys Crystals", Met. Trans.. 2 (1971), 215-219

[2] J. K. Tien and R. P. Gamble, "The Influence of applied Stress and Stress Sense on Grain Boundary Precipitate Morphology in a Nickel-base Superalloy During Creep", Met. Trans., 2 (1971), 1663-1667

[3] J. K. Tien and R. P. Gamble, " Effects of Stress Coarsening on Coherent Particle Strengthening", Met. Irans., 3 (1972), 2157-2162
[4] T. Miyazaki, K. Nakamura, and H. Mori, "Experimental and Theoretical investigations on morphological changes of $\gamma^{\prime}$ Precipitates in Ni-Al single Crystals During Uniaxial Stress-Annealing", J. Mat. Sci., $14(1979), 1827-1837$

[5] A. Pineau, "Influence of Uniaxial Stress on the morphology of Coherent Precipitates During Coarsening", Acta Metall., 24 (1976), 559-564

[6] S. Socrate and D. M. Parks, "Numerical Determination of the Elastic Driving Force for Directional Coarsening in Ni-Superalloys", Acta Metall. Mater, 41 (1993), 2185-2209

[7] C. Carry and J. L. Strudel, "Apparent and Effective Creep parameters in Single Crystals of a Nickel Base Superalloy- II Secondary Creep", Acta Metall., 26 (1978), 859-870

[8] T. M. Pollock and A. S. Argon, "Directional Coarsening in Nickel-Based Single Crystals with High Volume Fractions of Coherent Precipitates", Acta Metall. Mater., 42 (1994), 1859-1874

[9] M. Véron, Y. Bréchet, F. Louchet, "Directional Coarsening of $\gamma$ ' Precipitates in Nickel Based Superalloys : Driving Force and Influence of a Prestrain", Phil. Mag. Letters, (1996), (in press)

[10] A. Royer et al., "Temperature Dependence of the Lattice Mismatch of the AM1 Superalloy. Influence of the $\gamma$ ' Precipitates Morphology", Phil. Mag. A, 72 (1995), 669-689

[11] J. Y. Buffière and M. Ignat, "Dislocation Based Criterion for the Raft Formation in Nickel-Based Superalloys Single Crystals", Acta Metall. Mater., 43 (1995), 1791-1797

[12] A. Fredholm, "Monocristaux d'Alliages Base Nickel. Rclation entre composition, Microstructure et Comportement en Fluage à haute température", ( $\mathrm{PhD}$ thesis, Ecole Nationale des Mines de Paris, 1987).

[13] M. Véron and P. Bastie,"Strain Induced Directional Coarsening in Nickel Based Superalloy. Investigation on Kinetics Using SANS Technique", Scripta Metall. Mater., submitted

[14] M. Véron, Y. Bréchet, F. Louchet , "Directional Coarsening of Nickel Based Superalloys : Computer Simulation at Mesoscopic Scale", Acta Metall. Mater. (1996), in press 
[15] J. Philibert, "Diffusion et transport de matiere dans les solides", (Les Ulis Cedex, France; Editions de Physique, 1985), 13.

[16] A. Ardell, " The Effect of Volume fraction on Particle Coarsening: Theoretical Considerations", Acta Metall., 20 (1972), 61-71

[17] E. Nembach and G. Neite, "Precipitation Hardening of Superalloys by Ordered $\gamma$ '-Particles", Prog. Mater. sci., $29(1985), 177-319$

[18] P. Caron and T. Khan, "Improvement of Creep Strength in a Nickel base Single-Crystal Superalloy by Heat Treatment", Mat. Sci. Eng., 61 (1983) 173-184

[19] J. Courbon and F. Louchet, "On the Mechanism of Formation of Superlattice Stacking Faults in L12 Precipitates Embedded in a F.C.C Matrix", Phys. Stat. Sol. (a), 137 (1993), 417-428

[20] L.P. Kubin, B. Lisiecki and P. Caron, "Octahedral slip instabilities in $\gamma / \gamma$ ' superalloy single crystals CMSX2 and AM3", Phil. Mag. A, 71 (1995),991-1009

\section{Appendix :}

Compatibility of strain rate assuming no deformation of $\gamma^{\prime}$, leads to :

$$
1\left(\sigma_{\mathrm{V}}\right)^{\mathrm{n}}=\mathrm{w}_{\mathrm{h}}\left(\sigma_{\mathrm{h}}\right)^{\mathrm{n}}
$$

and the partitioning of the applied stress gives :

$$
\sigma_{\mathrm{v}}\left[\left(1+w_{\mathrm{V}}\right)^{2}-\mathrm{l}^{2}\right]+\sigma_{\mathrm{h}} \mathrm{l}^{2}=\sigma_{\mathrm{a}}\left(1+\mathrm{w}_{\mathrm{v}}\right)^{2}
$$

where $l$ is the mean size of the precipitates, $\sigma$ and $w$ are the stress and the width of a channel; the ' $\mathrm{v}$ ' indices denotes a vertical one, whereas ' $h$ ' denotes a horizontal one; $n$ is the Norton's law coeflicient of the matrix.

The relation between $\sigma$ and $\varepsilon$ in the $\gamma$ channels has been assumed to follow Norton's law :

$$
\varepsilon=\mathrm{K} \sigma^{\mathrm{n}}
$$

The capillary energy density driving this process is given by :

$$
\mathrm{E}_{\mathrm{i}}=2 \Gamma / \mathrm{w}
$$

where $\Gamma$ is the $\gamma / \gamma^{\prime}$ interfacial energy, and $w$ is the width of the channel (horizontal or vertical). 